\title{
2-LOCAL $*$-LIE AUTOMORPHISMS OF SEMI-FINITE FACTORS
}

\author{
XiaOchun FAng, XingPeng ZhaO AND Bing YANG
}

\begin{abstract}
Let $\mathscr{M}$ be a semi-finite von Neumann algebra factor on a complex Hilbert space $H$ with dimension greater than 3 . Then every surjective 2-local $*$-Lie automorphism $\Phi$ of $\mathscr{M}$ is of the form $\Phi=\Psi+\tau$, where $\Psi$ is a $*$-automorphism or the negative of a $*$-anti-automorphism of $\mathscr{M}$, and $\tau$ is a mapping from $\mathscr{M}$ into $\mathbb{C} I$ vanishing on every sum of commutators.
\end{abstract}

Mathematics subject classification (2010): 47B49.

Keywords and phrases: Factor, $*$-Lie automorphism, 2-local $*$-Lie automorphism.

\section{REFERENCES}

[1] R. V. KADison, Local derivations, J. Algebra. 130 (1990), no. 2,494-509.

[2] D. R. LARson, A. R. Sourour, Local derivations and local automorphisms of B(X), Proc. Sympos. Pure Math. 51 (1990),187-194.

[3] R. L. CRIST, Local derivations on operator algebras, J. Funct. Anal. 135 (1996), no. 1, 76-92.

[4] A. B. A. Essaleh, A. M. Peralta, M. I. Ramírez, Weak-local derivations and homorphisms on $C^{*}$-algebras, Linear Multilinear Algebra. 64 (2016), no. 2, 169-186.

[5] W. Jing, Local derivations of reflexive algebras, Proc. Am. Math. Soc. 125 (1997), no. 3, 869-873.

[6] B. E. Johnson, Local derivations on $C^{*}$-algebras are derivations, Trans. Am. Math. Soc. 353 (2000), no.1, 313-325.

[7] S. Kim, J. Kim, Local automorphisms and derivations on $M_{n}$, Proc. Am. Math. Soc. 132 (2004), 1389-1392.

[8] L. Molnar, Local automorphisms of operator algebras on Banach space, Proc. Am. Math. Soc. 131 (2003), no. 6, 1867-1874.

[9] P. ŠEMrL, Local automorphisms and derivations on B(H), Proc. Am. Math. Soc. 125 (1997), no. 9, 2677-2680.

[10] S. Ayupov, K. Kudaybergenov, 2-local derivations and automorphisms on B(H), J. Math. Anal. Appl. 395 (2012), no. 1, 15-18.

[11] S. Ayupov, K. Kudaybergenov, 2-local derivations on von Neumann algebras, Positivity, 19 (2015), no. 3, 445-455.

[12] S. Ayupov, K. Kudaybergenov, I. Rakhimov, 2-local derivations on finite dimensional Lie algebras, Linear Algebra Appl. 474 (2015), no. 1, 1-11.

[13] J. C. CABello, A. M. Peraita, Weak-2-local symmetric maps on $C^{*}$-algebras, Linear Algebra Appl. 494 (2016), no. 2,32-43.

[14] L. Liu, T. Wong, A note on 2-local maps, Proc. Edinb. Math. Soc. 49 (2006), no.3, 701-708.

[15] L. LiU, 2-local Lie derivations on semi-finite factor von Neumann algebras, Linear and Multilinear Algebra. 64 (2016), no. 9, 1679-1686.

[16] L. CHen, L. HuAng, F. LU, 2-local Lie isomorphism of operator algebras on Banach spaces, Studia Math. 229 (2015), no. 1, 1-11.

[17] Z. BAI, S. DU, Multiplicative *-Lie isomorphism between factors, J. Math. Anal. Appl. 346 (2008), no. $1,327-335$.

[18] Z. BAI, S. DU, Multiplicative *-Lie isomorphism between von Neumann algebras, Linear Multilinear Algebra. 60 (2012), no. 3, 311-322.

[19] C. J. LI, F. Y. LU, 2-local *-Lie isomorphisms of operator algebras, Aequat. Math. 90 (2016), no. 5, 905-916. 
[20] W. S. Mardindale III, Lie isomophisms of prime rings, Trans. Am. Math. Soc. 142 (1969), $437-$ 455.

[21] R. V. Kadison, J. R. Ringrose, Fundamentals of the theory of operator algebras, Volume II, Pure and applied mathematics. 1986.

[22] C. R. Miers, Commutativity preserving maps of factors, Can J Math. 40 (1988), no. 1, 248-256. 